\title{
THE EVOLUTION OF A TOBACCO SMOKING CESSATION AND HARM REDUCTION PROGRAMME IN A PSYCHIATRIC HOSPITAL
}

\author{
I. Ganhao', M. Trigo' ${ }^{1}$ A. Paixao' ${ }^{1}$ \\ ${ }^{1}$ Centro Hospitalar Psiquiatrico de Lisboa, \\ Tobacco Smoking Cessation and Harm Reduction Programme, Lisbon, Portugal
}

\section{Objectives:}

To present and reflect on a tobacco smoking cessation and harm reduction programme in a psychiatric hospital in Lisbon over a ten year period (2009-2019).

Aims:

Tobacco smoking is one of the major modifiable behaviours to enhance overall health and lower morbidity and mortality. People who suffer from mental illness, especially severe mental illness, are more likely to smoke, start at a younger age, be heavy smokers, have less access to treatment, make fewer quit attempts and relapse more frequently.

Interventions aimed at changing tobacco smoking, like other behavioural changes, are regarded as challenging, time and energy consuming and results difficult to define and quantify.

\section{Materials and Methods:}

The programme has maintained the same team over these 10 years (two psychologists and one psychiatrist) and has consisted of:

1. Group intervention strategies initially implemented for clinically stable outpatients.

2. Patients from the rehabilitation, residential and forensic units were included in the group with outpatients.

3. Another group intervention was started on a mixed rehabilitation and residential unit (2013-2017).

4. The programme was extended to acute general psychiatric inpatient wards from 2015 onwards, including an alcohol treatment unit and more recently an adolescent and young adult ward (patients aged 15 to 25 years old).
The programme reached 1181 outpatients and 861 inpatients.

Strategies for promoting change evolved from traditional psychological and pharmacological to integrating interventions facilitated by play, art and group dynamics.

In the spirit of play, team spirit, and promoting healthy lifestyles for people with mental health illnesses and the resilience of those who care for them, figure 1 represents the results as to smoking cessation, smoking reduction and dropouts of the patients attending the outpatient group during April and May 2019 (total of 8 sessions; 32 patients).

\section{Results and Conclusions:}

This tobacco smoking treatment programme in a psychiatric setting has the longest duration and greatest diversity of interventions that we know of in Portugal.

In spite of challenges, the programme has survived and expanded its activity. The team has learned to creatively adapt intervention strategies, tolerate frustration, understand and defend that measures of success expand far beyond tobacco smoking cessation rates to harm reduction and better quality of life in other domains.

Interventions evolved to include substance abuse, especially cannabinoids, caffeine consumption, weight control and promoting physical activity and other healthy lifestyle behaviours.

"Start where you are. Use what you have. Do what you can."

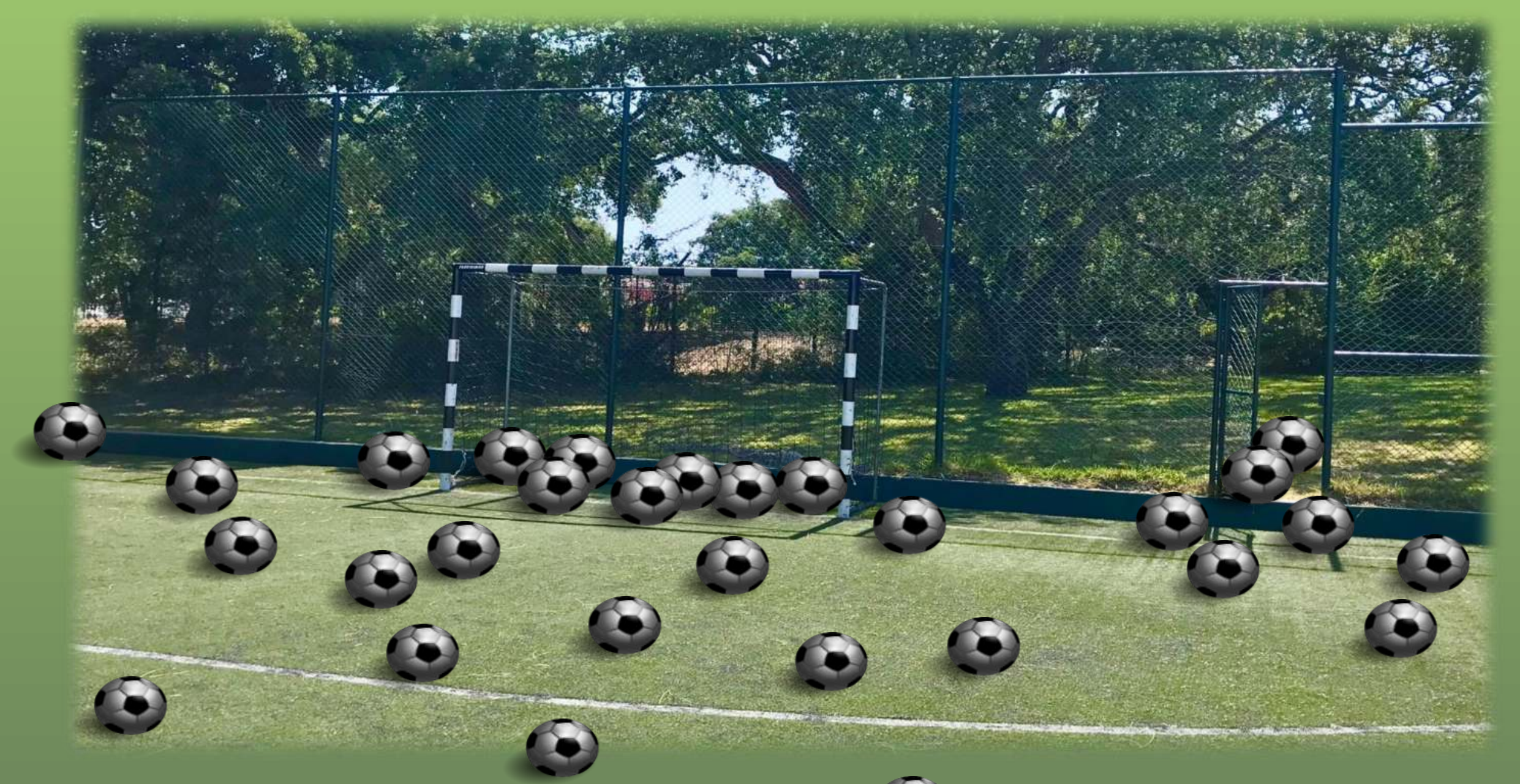

Figure 1: 32 participants in programme during April and May 2019

Balls in net=smoking cessation $(21,8 \%)$

Balls looking at net inside photo $=$ smoking reduction $(37,5 \%)$

Balls beyond or on borders of photo=contemplators $(18,7 \%)$

Balls leaving the field=the right to give up at the time but the door is always open $(21,8 \%)$ 